\title{
Effect of the Combination of Creatine Monohydrate Plus HMB Supplementation on Sports Performance, Body Composition, Markers of Muscle Damage and Hormone Status: A Systematic Review
}

\author{
Julen Fernández-Landa ${ }^{1}{ }^{\circledR}$, Julio Calleja-González ${ }^{1}$, Patxi León-Guereño ${ }^{2}{ }^{\circledR}$, \\ Alberto Caballero-García ${ }^{3}$, Alfredo Córdova ${ }^{4}$ and Juan Mielgo-Ayuso ${ }^{4, *(D)}$ \\ 1 Laboratory of Human Performance, Department of Physical Education and Sport, Faculty of Education, \\ Sport Section, University of the Basque Country, 01007 Vitoria, Spain; julenfdl@hotmail.com (J.F.-L.); \\ julio.calleja.gonzalez@gmail.com (J.C.-G.) \\ 2 Faculty of Psychology and Education, University of Deusto, Campus of Donostia-San Sebastián, \\ 20012 San Sebastián, Spain; patxi.leon@deusto.es \\ 3 Department of Anatomy and Radiology, Faculty of Health Sciences, University of Valladolid. Campus de \\ Soria, 42003 Soria, Spain; albcab@ah.uva.es \\ 4 Department of Biochemistry, Molecular Biology and Physiology, Faculty of Health Sciences, University of \\ Valladolid, 42003 Soria, Spain; a.cordova@bio.uva.es \\ * Correspondence: juanfrancisco.mielgo@uva.es; Tel.: +34-975-129-187
}

Received: 13 September 2019; Accepted: 18 October 2019; Published: 20 October 2019

\begin{abstract}
Although there are many studies showing the isolated effect of creatine monohydrate $(\mathrm{CrM})$ and $\beta$-hydroxy $\beta$-methylbutyrate (HMB), it is not clear what effect they have when they are combined. The main purpose of this systematic review was to determine the efficacy of mixing CrM plus HMB in comparison with their isolated effects on sports performance, body composition, exercise induced markers of muscle damage, and anabolic-catabolic hormones. This systematic review was carried out in accordance with PRISMA (Preferred Reporting Items for Systematic Reviews and Meta-Analyses) statement guidelines and the PICOS model, for the definition of the inclusion criteria. Studies were found by searching PubMed/MEDLINE, Web of Science (WOS), and Scopus electronic databases from inception to July 3rd 2019. Methodological quality and risk of bias were assessed by two authors independently, and disagreements were resolved by third-party evaluation, in accordance with the Cochrane Collaboration Guidelines samples. The literature was examined regarding the effects of the combination of CrM plus HMB on sport performance using several outcome variables (athletic performance, body composition, markers of muscle damage, and hormone status). This systematic review included six articles that investigated the effects of CrM plus HMB on sport performance (two on strength performance, showing improvements in one of them; three on anaerobic performance, presenting enhancements in two of them; and one on aerobic performance, not presenting improvements), body composition (three on body mass, showing improvements in one of them; two on fat free mass, presenting increases in one of them; and two on fat mass, showing decreases in one of them) and markers of muscle damage and hormone status (four on markers of muscle damage and one on anabolic-catabolic hormones, not showing benefits in any of them). In summary, the combination of 3-10 g/day of CrM plus $3 \mathrm{~g} /$ day of HMB for 1-6 weeks could produce potential positive effects on sport performance (strength and anaerobic performance) and for 4 weeks on body composition (increasing fat free mass and decreasing fat mass). However, this combination seems to not show positive effects relating to markers of exercise-induced muscle damage and anabolic-catabolic hormones.
\end{abstract}


Keywords: sport nutrition; anaerobic; aerobic; body composition; muscle recovery

\section{Introduction}

Supplements and sport foods may help to prevent or treat nutrition deficiencies, and occasionally have a direct ergogenic effect [1]. However, there are few supplements supported by strong evidence that produce a significant effect on sports performance [1]. In this sense, while there are several supplements that have strong scientific evidence for use in sports-specific situations using evidence-based protocols, such as creatine monohydrate $(\mathrm{CrM})$, others-although deserving of further research-require more scientific support; for example, $\beta$-hydroxy $\beta$-methylbutyrate (HMB) [2].

$\mathrm{CrM}$ is one of the most popular performance supplements used by athletes that may promote aerobic [3,4] and anaerobic performance [5], strength [6-9], body composition [6,8,10], reduced markers of exercise induced muscle damage, and anabolic-catabolic hormones [11,12]. The main action of CrM is to increase the muscle creatine (CR) stores to replace adenosine triphosphate (ATP) degradation during exercise [13]. Moreover, it increases the muscle glycogen pool by stimulating muscle glycogen synthesis based on the augmentation of muscle cells and muscle CR content [14,15]. Additionally, this effect could increase creatine-phosphocreatine (Cr-PCr) shuttling, improving aerobic capacity [16]. Furthermore, CR supplementation increases lean tissue mass and upper and lower body muscular strength [17].

HMB could also enhance sports performance in terms of aerobic power and capacity [18-20], anaerobic capacity [18,20], strength [21-24], body composition [18,25], markers of muscle damage [26], and hormone status [25]. The main role of HMB is to stimulate muscle protein synthesis by an up-regulation of Mammalian Target of Rapamycin kinase (mTOR) [27]. Additionally, HMB could augment muscle glycogen storage $[28,29]$ and can increase gene expression of peroxisome proliferator-activated receptor gamma co-activator 1-alpha $\alpha$ (PGC-1 $\alpha)$, enhancing mitochondrial biogenesis, and hence oxidative function, to enhance aerobic capacity [30]. Moreover, this benefit in sports performance could be motivated by an augmented lean body mass (LBM) and/or fat free mass (FFM) $[20,22,31]$, and reduced fat mass (FM) $[18,20,31]$.

Given that these two supplements have different physiological pathways to improve performance [13-15,28,29,32-34], it could be assumed that the combination of both complements would improve sports performance compared to taking them alone. Therefore, some authors have considered the utilization of both supplements together (CrM plus HMB) with the aim of producing an additive or synergistic effect. To the best of the authors' knowledge, the results of the studies investigating combined supplementation are not clear. Some studies show possible improvements in performance [35-37], body composition (increases FFM and decreases FM) [36,37], markers of muscle damage [36], and hormones status [38], but others found no changes in these outcomes [38-40]. Therefore, the main purpose of this systematic review was to determine the efficacy of mixing CrM plus HMB in improving sports performance, body composition (increases FFM, LBM, and decreases FM), markers of exercise induced muscle damage, and anabolic-catabolic hormones in comparison with their isolated effects.

\section{Methods}

\subsection{Literature Search Strategies}

This systematic review was carried out in accordance with PRISMA ${ }^{\circledR}$ (Preferred Reporting Items for Systematic Reviews and Meta-Analyses) statement guidelines and the PICOS model for the definition of the inclusion criteria: P (Population): "athletes", I (Intervention): "impact of the combination of HMB and CrM in sport", C (Comparators): "same conditions with control, placebo, only HMB or only $\mathrm{CrM}^{\prime}, \mathrm{O}$ (Outcome): "sport performance, body composition, markers of muscle damage and hormone 
status", and S (study design): "clinical trial" [41]. A systematic search of the current scientific literature was undertaken for studies that investigated the mixed supplementation of CrM plus HMB in sports performance and recovery. Studies were found by searching PubMed/MEDLINE, Web of Science (WOS), and Scopus from inception to July 3rd 2019, using the following Boolean search equation: ("creatine monohydrate supplementation"[All Fields] OR "creatine supplementation"[All Fields]) AND ("HMB supplementation"[All Fields] OR "beta hydroxy beta methylbutyrate supplementation"[All Fields] OR (beta-Hydroxy[All Fields] AND methylbutyrate[All Fields] AND supplementation[All Fields])) AND ("muscle damage" [All Fields] OR "hormone status"[All Fields] OR ("athletes"[MeSH Terms] OR "athletes"[All Fields]) OR ("exercise"[MeSH Terms] OR "exercise"[All Fields]) OR "sport performance"[All Fields] OR "body composition"[All Fields]). Through this equation, relevant articles in this field were obtained by applying the snowball strategy. All titles and abstracts from the search were cross-referenced to identify duplicates and any potential missing studies. The titles and abstracts were screened for a subsequent full-text review. The search for published studies was independently performed by two authors (J.F.L. and J.M.A.), and disagreements about all outcomes were resolved through discussion.

\subsection{Inclusion and Exclusion Criteria}

For the articles obtained in this search, the following inclusion criteria were applied to select studies: (1) a well-designed experiment that included ingestion of the combination of CrM plus HMB; (2) with an identical experimental situation related to the ingestion of a placebo, CrM only, and/or HMB only; (3) testing the effects of mixed supplementation on sports performance, body composition, markers of muscle damage, and/or hormone status; (4) clinical trial; (5) with clear information regarding the administration of ergogenic aids (dosage and timing); and (6) published in any language. The following exclusion criteria were applied to the experimental protocols of the investigation: (1) supplementation was mixed with other supplements or was a multi-ingredient compound; (2) carried out in participants with a previous condition, injury, or health problems. There were no filters applied to the athletes' level, gender, ethnicity, or age to increase the analytic power of the analysis.

Once the inclusion/exclusion criteria were applied to each study, data on study source (including authors and year of publication), study design, supplement administration (dose and timing), sample size, characteristics of the participants (level, race and gender), and final outcomes of the interventions were extracted independently by two authors (J.F.L. and J.M.A.) using a spreadsheet (Microsoft Inc, Seattle, WA, USA). Subsequently, disagreements were resolved through discussion until a consensus was reached, or by third-party adjudication (J.C.G.).

\subsection{Study Selection}

One reviewer (J.F.L.) searched the databases and selected the studies. A second reviewer (J.M.A.) was available to help with study eligibility. No disagreements about the appropriateness of an article were encountered.

\subsection{Outcome Measures}

The literature was examined regarding the effects of the combination of CrM plus HMB in sports performance using several outcome variables, such as athletic performance [35-37,39,40], body composition [36,37,40], markers of muscle damage [35-39], and hormone status [38].

\subsection{Quality Assessment of the Experiments}

Methodological quality and risk of bias were assessed by two authors independently (J.F.L. and J.M.A.), and disagreements were resolved by third-party evaluation (J.C.G.), in accordance with the Cochrane Collaboration Guidelines [42]. The items on the list were divided into six domains: selection bias (random sequence generation, allocation concealment); performance bias (blinding of participants and researchers); detection bias (blinding of outcome assessment); attrition bias (incomplete outcome 
data); reporting bias (selective reporting); and other types of bias. For each research paper, domains were judged by consensus (J.F.L., J.M.A.), or third-party adjudication (J.C.G.). They were characterized as 'low' if criteria for a low risk of bias were met (plausible bias unlikely to seriously alter the results) or 'high' if criteria for a high risk of bias were met (plausible bias that seriously weakens confidence in the results), or it was considered 'unclear' (plausible bias that raises some doubt about the results), if the risk of bias was unknown. Full details are given in Figures 1 and 2.

\begin{tabular}{|c|c|c|c|c|c|c|c|}
\hline & 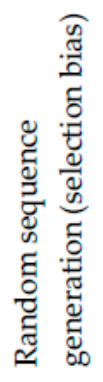 & 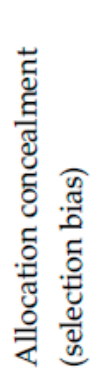 & 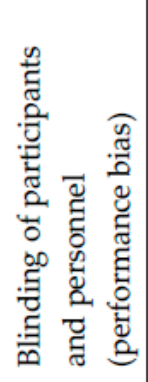 & 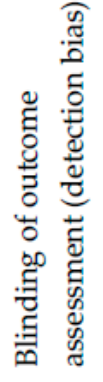 & 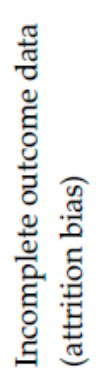 &  & $\begin{array}{l}\frac{\mathscr{g}}{0} \\
\stackrel{0}{0} \\
\tilde{\Xi} \\
0\end{array}$ \\
\hline Crowe et al., 2003 [38] & & $?$ & & & $\odot$ & 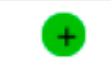 & \\
\hline Faramarzi et al., 2009 [35] & + & $?$ & + & $?$ &  & + & \\
\hline Jowko et al., 2001 [36] &  & $?$ & + & 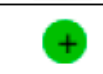 & 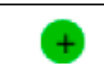 & + & 1 \\
\hline O,Connor et al., 2003 [39] & & $?$ & & &  &  & \\
\hline O,Connor et al., 2007 [40] & & $?$ & & & + & 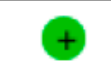 & \\
\hline Zajac et al., 2003 [37] & & $?$ & $?$ & $?$ & + & + & \\
\hline
\end{tabular}

Figure 1. Risk of bias graph: review authors' judgments about each risk of bias item presented as percentages across all studies. $\odot$ Indicates low risk of bias; ? indicates unknown risk of bias;

indicates high risk of bias.

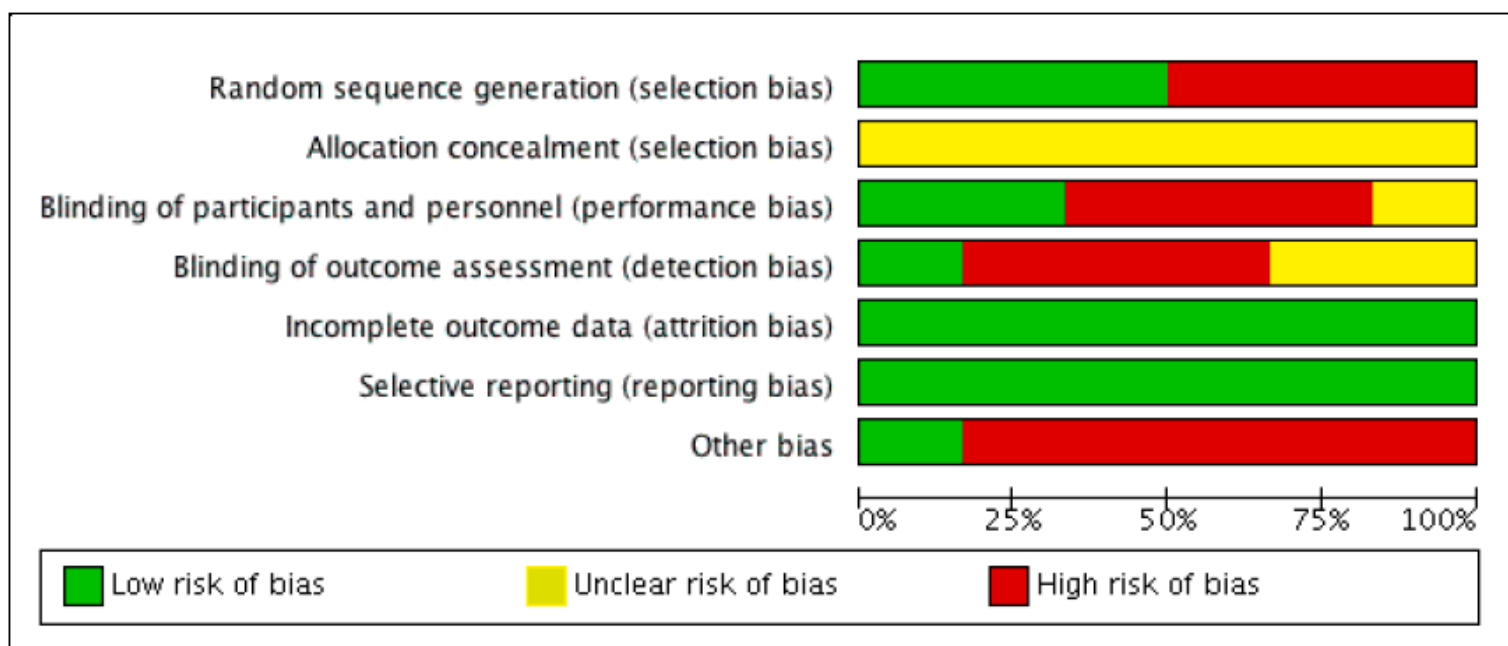

Figure 2. Risk of bias summary: review authors' judgments about each risk of bias item for all studies.

\section{Results}

\subsection{Main Search}

A search of electronic databases revealed seventeen relevant studies, with an additional three studies found by searches in reference lists (Figure 3). After removing duplicate studies $(n=14)$ and 
screening titles and abstracts $(n=6)$, eight studies were retained [35-40,43,44]. Following full-text screening, only two studies were excluded [43,44] (one was combined with a non-usual training [43] and the other one was performed with only one of the supplements [44]). Thereby, six studies were included for this systematic review [35-40].

The design of the six studies included one randomized, double-blind, placebo-controlled study [36], two randomized, placebo-controlled studies [35,37], and three controlled studies [38-40]. Five out of six studies were conducted in intermittent team sports: three in rugby [38-40], one in basketball [37], and one in soccer [35]. The last study was carried out on healthy males [36]. The sum of all study participants included in this review were 201 males, with 161 being participants in high-level sports leagues (five studies) [35,37-40] and the remaining 40 being moderately trained participants [36].
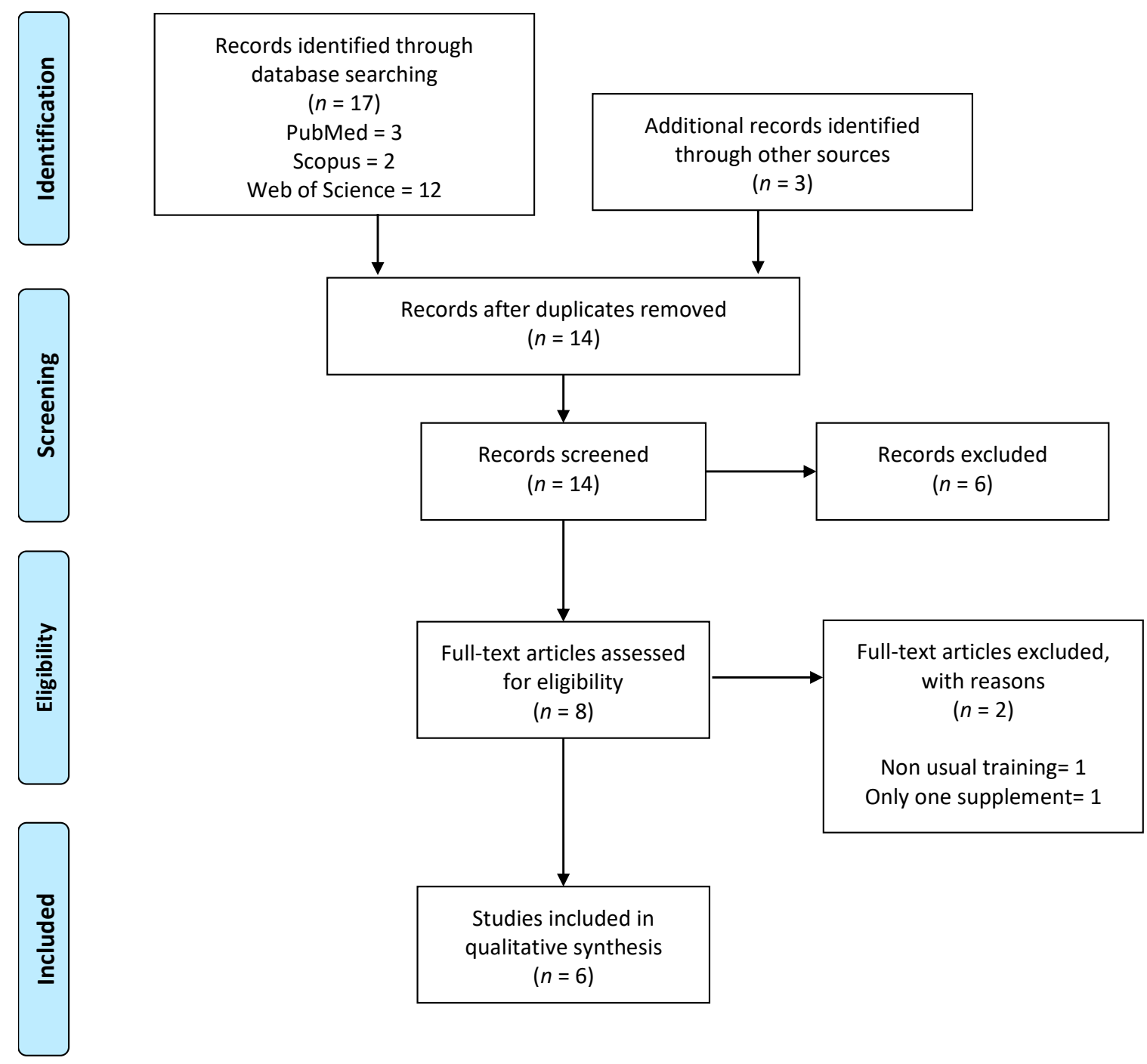

Figure 3. Preferred Reporting Items for Systematic Reviews and Meta-Analyses (PRISMA) flow diagram.

Some studies divided the participants into four different supplementation groups: placebo group (PLAG) or control group (CON), creatine group (CrMG), HMB group (HMBG), and CrM plus HMB group (CrM/HMBG) $[36,37]$. Other studies assigned participants to three groups: PLAG or CON, HMBG, and CrM/HMBG [35,38-40]. 


\subsection{CrM and HMB Supplementation}

The duration of the interventions in the studies was between six days and six weeks (42 days). In four of the studies included in this systematic review the supplementation protocol consisted of $3 \mathrm{~g} /$ day of CrM and $3 \mathrm{~g} /$ day of HMB [35,38-40]. The exceptions were the studies by Jowko et al. [36] and Zajac et al. [37], in which the CrM supplementation consisted of $20 \mathrm{~g} /$ day for the first 7 days followed by $10 \mathrm{~g} /$ day the next 14 days, and $15 \mathrm{~g} /$ day the first 5 days followed by $5 \mathrm{~g} /$ day the next 25 days, respectively. Moreover, the training program frequency performed by the athletes was 3 or 4 days per week [36-40] or 6 days per week [35] in a resistance training program.

\subsection{Sports Performance Outcomes}

Table 1 presents the different tests carried out to determine the performance measured outcomes by running an anaerobic speed test (RAST) [35], one repetition maximum (1-RM) test of different strength exercises [36], multistage aerobic capacity test, cycling maximally for 60 seconds [39], muscular strength, muscular endurance, leg power tests [40], and a triple Wingate test [37].

The combination of CrM plus HMB showed improvements in strength performance in one study [36], but the other study that measured this parameter did not find performance changes among groups [40]. On the other hand, anaerobic capacity was enhanced in two investigations [36,37] when the athletes ingested CrM plus HMB, although the study by O'Connor et al. [39] did not find any improvement. The last performance variable was aerobic capacity, measured by a multistage aerobic capacity test, and it was unchanged in all groups [39].

\subsection{Body Composition Outcomes}

Table 2 shows the body composition measures. Zajac et al. [37] found reductions in FM (regarding CON/PLG and CrMG) in basketball players when they were supplemented with CrM plus HMB, although Jowko et al. [36] did not. On the other hand, Zajac et al. found an increase in BM and FFM (regarding CON/PLG and HMBG), contrasting to studies by Jowko et al. [36] and O'Connor et al. [40], who did not find changes in this parameter among groups when CrM plus HMB was ingested.

\subsection{Markers of Muscle Damage and Hormone Status Outcomes}

Table 3 displays muscular blood isoenzymes, such as creatine kinase (CK) [35,36,38], lactate dehydrogenase (LDH) [35], and blood lactate concentration (LA) [37,39], which were unchanged after supplementation with CrM plus HMB. Moreover, anabolic/catabolic blood hormones (testosterone and cortisol) did not show changes when the athletes were supplemented with CrM plus HMB [36]. 
Table 1. Summary of studies included in the systematic review that investigated the effect of CrM plus HMB on athletic performance abilities.

\begin{tabular}{|c|c|c|c|c|c|c|}
\hline \multirow{2}{*}{ Author/s } & \multirow{2}{*}{ Population } & \multirow{2}{*}{ Intervention } & \multirow{2}{*}{ Outcomes } & \multicolumn{3}{|c|}{ Effects } \\
\hline & & & & CrM+HMB Vs CON/PLG & CrM+HMB Vs CrMG & CrM+HMB Vs HMBG \\
\hline $\begin{array}{l}\text { Faramarzi et al., } \\
\quad(2009) \text { [35] }\end{array}$ & $\begin{array}{l}24 \text { soccer players } \\
(21.6 \pm 0.1 \text { years })\end{array}$ & $\begin{array}{c}\text { Randomized, placebo- } \\
\text { controlled } \\
\text { CrM: } 3 \text { g/day } \\
\text { HMB: } 3 \text { g/day } \\
\text { Duration: } 6 \text { days }\end{array}$ & $\begin{array}{ll}\text { - } & \text { Peak Power (RAST) } \\
\text { - } & \text { Mean Power (RAST) } \\
& \text { Fatigue Index (RAST) }\end{array}$ & $\begin{array}{ll}\text { - } & \uparrow \text { Peak Power } \\
\text { - } & \uparrow \text { Mean Power } \\
\text { - } & \bigoplus \text { Fatigue Index }\end{array}$ & No data shown & $\begin{array}{l}\text { - } \quad \uparrow \text { Peak Power } \\
\text { - } \quad ⿴ \text { Mean Power } \\
\text { - } \quad ⿴ \text { Fatigue Index }\end{array}$ \\
\hline $\begin{array}{l}\text { Jówko et al., } \\
\text { (2001) [36] }\end{array}$ & $\begin{array}{l}40 \text { healthy males } \\
(21.0 \pm 2.1 \text { years })\end{array}$ & $\begin{array}{c}\text { Randomized, double-blind, } \\
\text { placebo-controlled } \\
\text { CrM: } 20 \text { g/day (first } 1 \text { week) + } \\
10 \text { g/day ( } 2 \text { weeks) } \\
\text { HMB: } 3 \text { g/day } \\
\text { Duration: } 3 \text { weeks }\end{array}$ & $\begin{array}{l}\text { - Accumulative strength } \\
\text { tests (1-RM) }\end{array}$ & $\begin{array}{ll}- & \uparrow \text { Accumulative } \\
\text { strength tests (1-RM) }\end{array}$ & $\begin{array}{l}\text { - } \quad \text { Accumulative } \\
\text { strength tests (1-RM) }\end{array}$ & $\begin{array}{l}\text { - } \quad \text { Accumulative } \\
\text { strength tests (1-RM) }\end{array}$ \\
\hline $\begin{array}{l}\text { O'Connor \& Crowe } \\
\text { (2003) [39] }\end{array}$ & $\begin{array}{l}27 \text { male elite rugby } \\
\text { players } \\
\text { (18-32 years) }\end{array}$ & $\begin{array}{c}\text { Controlled } \\
\text { CrM: } 3 \text { g/day } \\
\text { HMB: } 3 \text { g/day } \\
\text { Duration: } 6 \text { weeks }\end{array}$ & $\begin{array}{l}\text { - Aerobic performance } \\
\text { (multistage aerobic } \\
\text { capacity test) } \\
\text { Anaerobic performance } \\
\text { (60 second maximal } \\
\text { anaerobic capacity test) }\end{array}$ & $\begin{array}{l}\text { - } \quad ⿴ \text { Aerobic capacity } \\
\text { - } \quad ⿴ \text { Anaerobic capacity }\end{array}$ & No data shown & $\begin{array}{l}\text { - } \quad ⿴ \text { Aerobic capacity } \\
\text { - } \quad ⿴ \text { Anaerobic capacity }\end{array}$ \\
\hline $\begin{array}{l}\text { O'Connor \& Crowe } \\
\text { (2007) [40] }\end{array}$ & $\begin{array}{c}30 \text { male elite rugby } \\
\text { players } \\
(24.9 \pm 1.5 \text { years })\end{array}$ & $\begin{array}{l}\text { Controlled } \\
\text { CrM: } 3 \text { g/day } \\
\text { HMB: } 3 \text { g/day } \\
\text { Duration: } \\
6 \text { weeks }\end{array}$ & 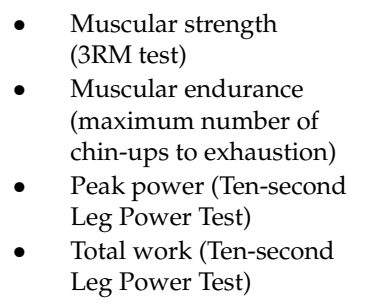 & 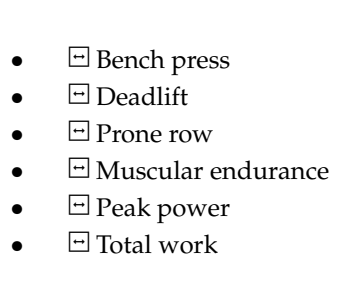 & No data shown & 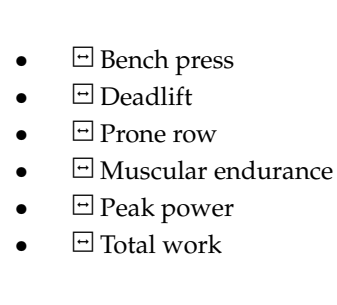 \\
\hline Zajac et al., (2003) [37] & $\begin{array}{c}52 \text { well trained } \\
\text { basketball players } \\
(25.6 \pm 5.6 \text { years })\end{array}$ & $\begin{array}{c}\text { Randomized, placebo- } \\
\text { controlled } \\
\text { CrM: } 15 \text { g/day (first } 5 \text { days) }+ \\
5 \text { g/day (rest of the days) } \\
\text { HMB: } 3 \text { g/day } \\
\text { Duration: } 30 \text { days }\end{array}$ & $\begin{array}{l}\text { - Relative maximal } \\
\text { anaerobic power (triple } \\
\text { Wingate test) } \\
\text { - Relative total work (triple } \\
\text { Wingate test) }\end{array}$ & $\begin{array}{l}\text { - } \quad \uparrow \text { Relative maximal } \\
\text { - } \quad \uparrow \text { Relative total work }\end{array}$ & $\begin{array}{l}\text { - } \quad \boxminus \text { Relative maximal } \\
\text { - } \quad \boxminus \text { Relative total work }\end{array}$ & $\begin{array}{l}\text { - } \quad \uparrow \text { Relative maximal } \\
\text { - } \quad \uparrow \text { Relative total work }\end{array}$ \\
\hline
\end{tabular}

CrM: Creatine monohydrate supplementation, HMB: HMB supplementation, CON/PLG: Placebo or control group, HMBG: HMB supplementation group, CrMG: Creatine monohydrate

supplementation group, RAST: Running Anaerobic Speed Test; $\uparrow:$ Increase, $\downarrow$ : Decrease, $\boxminus$ : No effect. 
Table 2. Summary of studies included in the systematic review that investigated the effect of CrM plus HMB on body composition.

\begin{tabular}{|c|c|c|c|c|c|c|}
\hline \multirow{2}{*}{ Author/s } & \multirow{2}{*}{ Population } & \multirow{2}{*}{ Intervention } & \multirow{2}{*}{ Outcomes } & \multicolumn{3}{|c|}{ Effects } \\
\hline & & & & CrM+HMB Vs CON/PLG & CrM+HMB Vs CrMG & CrM+HMB Vs HMBG \\
\hline $\begin{array}{l}\text { Jówko et al., } \\
\text { (2001) [36] }\end{array}$ & $\begin{array}{l}40 \text { healthy males } \\
(21.0 \pm 2.1 \text { years })\end{array}$ & $\begin{array}{c}\text { Randomized, double-blind, } \\
\text { placebo-controlled } \\
\text { CrM: } 20 \text { g/day (first } 1 \text { week) + } \\
10 \text { g/day ( } 2 \text { weeks) } \\
\text { HMB: } 3 \text { g/day } \\
\text { Duration: } 3 \text { weeks }\end{array}$ & $\begin{array}{ll}\text { - } & \mathrm{BM} \\
\text { - } & \mathrm{BF} \\
& \mathrm{LBM}\end{array}$ & $\begin{array}{ll}\text { - } & \text { ĐBM } \\
\text { - } & \text { ĐFM } \\
& \text { LBM }\end{array}$ & $\begin{array}{ll}\text { - } & \bigoplus \mathrm{BM} \\
\text { - } & \bigoplus \mathrm{FM} \\
\text { - } & \boxminus \mathrm{LBM}\end{array}$ & $\begin{array}{ll}\text { - } & \bigoplus \mathrm{BM} \\
\text { - } & \boxminus \mathrm{FM} \\
\text { - } & \boxminus \mathrm{LBM}\end{array}$ \\
\hline $\begin{array}{l}\text { O'Connor \& Crowe } \\
\text { (2007) [40] }\end{array}$ & $\begin{array}{c}30 \text { male elite rugby } \\
\text { players } \\
(24.9 \pm 1.5 \text { years })\end{array}$ & $\begin{array}{l}\text { Controlled } \\
\text { CrM: } 3 \text { g/day } \\
\text { HMB: } 3 \text { g/day } \\
\text { Duration: } \\
6 \text { weeks }\end{array}$ & $\begin{array}{ll}\text { - } & \text { BM } \\
\text { - } & \text { Sum of six skinfolds } \\
\text { - } & \text { Arm girth (relaxed) } \\
\text { - } & \text { Chest girth (fixed) } \\
\text { - } & \text { Waist girth } \\
\text { - } & \text { Hip girth } \\
\text { - } & \text { Thigh girth } \\
\text { - } & \text { Humur diameter diameter }\end{array}$ & 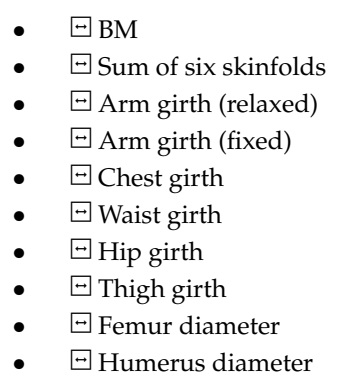 & No data showed & 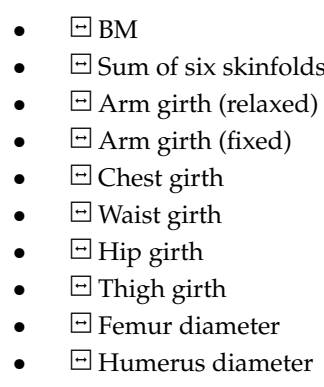 \\
\hline Zajac et al., (2003) [37] & $\begin{array}{c}52 \text { well trained } \\
\text { basketball players } \\
(25.6 \pm 5.6 \text { years })\end{array}$ & $\begin{array}{c}\text { Randomized, placebo- } \\
\text { controlled } \\
\text { CrM: } 15 \text { g/day (first } 5 \text { days) + } \\
5 \text { g/day (rest of the days) } \\
\text { HMB: } 3 \text { g/day } \\
\text { Duration: } 30 \text { days }\end{array}$ & $\begin{array}{ll}\text { - } & \text { BM } \\
\text { - } & \text { FFM } \\
\text { - } & \text { FM }\end{array}$ & $\begin{array}{ll}\bullet & \uparrow \mathrm{BM} \\
\bullet & \uparrow \mathrm{FFM} \\
\bullet & \downarrow \mathrm{FM}\end{array}$ & $\begin{array}{ll}\text { - } & \boxplus \mathrm{BM} \\
\bullet & \quad \mathrm{FFM} \\
\cdot & \downarrow \mathrm{FM}\end{array}$ & $\begin{array}{ll}\text { - } & \uparrow \mathrm{BM} \\
\text { - } & \uparrow \mathrm{FFM} \\
\text { - } & \oplus \mathrm{FM}\end{array}$ \\
\hline
\end{tabular}

CrM: Creatine monohydrate supplementation, HMB: HMB supplementation, CON/PLG: Placebo or control group, HMBG: HMB supplementation group, CrMG: Creatine monohydrate

supplementation group, LBM: Lean body mass, BM: Body mass, FM: Fat mass, FFM: Fat free mass; $\uparrow:$ Increase, $\downarrow$ : Decrease, $\bigoplus$ : No effect. 
Table 3. Summary of studies included in the systematic review that investigated the effect of CrM plus HMB on markers of muscle damage and hormone status outcomes.

\begin{tabular}{|c|c|c|c|c|c|c|}
\hline \multirow{2}{*}{ Author/s } & \multirow{2}{*}{ Population } & \multirow{2}{*}{ Intervention } & \multirow{2}{*}{ Outcomes } & \multicolumn{3}{|c|}{ Effects } \\
\hline & & & & CrM+HMB Vs CON/PLG & CrM+HMB Vs CrM & CrM+HMB Vs HMBC \\
\hline $\begin{array}{l}\text { Crowe et al., } \\
\text { (2003) [38] }\end{array}$ & $\begin{array}{c}28 \text { male elite rugby } \\
\text { players } \\
(24.9 \pm 0.7 \text { years })\end{array}$ & $\begin{array}{c}\text { Controlled } \\
\text { CrM: } 3 \text { g/day } \\
\text { HMB: } 3 \text { g/day } \\
\text { Duration: } 6 \text { weeks }\end{array}$ & $\begin{array}{ll}\text { - } & \text { Testosterone } \\
\text { - } & \text { Cortisol } \\
\text { - } & \text { CK } \\
\text { - } & \text { Urea }\end{array}$ &  & - No data shown &  \\
\hline $\begin{array}{l}\text { Faramarzi et al., } \\
\text { (2009) [35] }\end{array}$ & $\begin{array}{l}24 \text { soccer players } \\
(21.6 \pm 0.1 \text { years })\end{array}$ & $\begin{array}{c}\text { Randomized, placebo- } \\
\text { controlled } \\
\text { CrM: } 3 \text { g/day } \\
\text { HMB: } 3 \text { g/day } \\
\text { Duration: } 6 \text { days }\end{array}$ & $\begin{array}{ll}\text { - } & \mathrm{CK} \\
& \mathrm{LDH}\end{array}$ & $\begin{array}{l}\text { - } \quad \boxminus \mathrm{CK} \\
\text { - } \quad \mathrm{LDH}\end{array}$ & - No data shown & $\begin{array}{l}\text { • } \\
\text { • }\end{array}$ \\
\hline $\begin{array}{l}\text { Jówko et al., } \\
\text { (2001) [36] }\end{array}$ & $\begin{array}{l}40 \text { healthy males } \\
(21.0 \pm 2.1 \text { years })\end{array}$ & $\begin{array}{c}\text { Randomized, double-blind, } \\
\text { placebo-controlled } \\
\text { CrM: } 20 \text { g/day (first } 1 \text { week) + } \\
10 \text { g/day ( } 2 \text { weeks) } \\
\text { HMB: } 3 \text { g/day } \\
\text { Duration: } 3 \text { weeks }\end{array}$ & $\begin{array}{ll}\text { - } & \text { CK } \\
\text { - } & \text { Urea nitrogen }\end{array}$ & $\begin{array}{ll}\text { - } & \boxplus C K \\
& \downarrow \text { Urea nitrogen }\end{array}$ & $\begin{array}{ll}\text { - } & \uparrow C K \\
\text { - } & \text { ĐUrea nitrogen }\end{array}$ & $\begin{array}{ll}\text { - } & \uparrow C K \\
\text { - } & \oplus \text { Urea nitrogen }\end{array}$ \\
\hline $\begin{array}{c}\text { O'Connor \& Crowe } \\
\text { (2003) [39] }\end{array}$ & $\begin{array}{c}27 \text { male elite rugby } \\
\text { players } \\
\text { (18-32 years) }\end{array}$ & $\begin{array}{c}\text { Controlled } \\
\text { CrM: } 3 \text { g/day } \\
\text { HMB: } 3 \text { g/day } \\
\text { Duration: } 6 \text { weeks }\end{array}$ & - $\quad$ LA (blood) & - $\quad \boxminus \mathrm{LA}$ & - No data shown & - $\quad \boxminus \mathrm{LA}$ \\
\hline Zajac et al., (2003) [37] & $\begin{array}{c}52 \text { well trained } \\
\text { basketball players } \\
(25.6 \pm 5.6 \text { years })\end{array}$ & $\begin{array}{c}\text { Randomized, placebo- } \\
\text { controlled } \\
\text { CrM: } 15 \text { g/day (first } 5 \text { days) }+ \\
5 \text { g/day (rest of the days) } \\
\text { HMB: } 3 \text { g/day } \\
\text { Duration: } 30 \text { days }\end{array}$ & $\begin{array}{ll}- & \mathrm{LA} \\
- & \mathrm{CK} \\
- & \mathrm{LDH}\end{array}$ & $\begin{array}{l}\text { - } \quad \boxminus \mathrm{LA} \\
\text { - } \quad \boxminus \mathrm{CK} \\
\text { - } \quad \mathrm{LDH}\end{array}$ & $\begin{array}{ll}\text { - } & \boxplus \mathrm{LA} \\
\text { - } & \Xi \mathrm{CK} \\
\text { - } & \boxplus \mathrm{LDH}\end{array}$ & $\begin{array}{l}\text { - } \quad \text { - } \quad \uparrow \mathrm{LA} \\
\dot{\mathrm{CK}} \\
-\quad \uparrow \mathrm{LDH}\end{array}$ \\
\hline
\end{tabular}

CrM: Creatine monohydrate supplementation, HMB: HMB supplementation, CON/PLG: placebo or control group, HMBG: HMB supplementation group, CrMG: Creatine monohydrate supplementation group, CK: creatine kinase, LA: blood lactate, LDH: Lactate dehydrogenase; $\uparrow:$ Increase, $\downarrow$ : Decrease, $\bigoplus$ : No effect. 


\section{Discussion}

The main purpose of this systematic review was to summarize all scientific evidence about the effect of CrM plus HMB supplementation on variables related to physical performance (strength performance [36,40], anaerobic performance [35,37,39], aerobic performance [39]), body composition (LBM, BM, FM, FFM) [36,37,40], markers of muscle damage (CK, LDH, LA) [35-37,39], and hormone status (testosterone and cortisol) [38], as measured in the six studies included in this systematic review [35-40]. The main results indicated that the combination of 3-10 g/day of CrM plus HMB $3 \mathrm{~g} /$ day, over 1-6 weeks, could produce more improvements than taking them in an isolated way in strength performance, anaerobic performance, and for 4 weeks in body composition (increasing FFM and decreasing FM). However, no significant results were found on aerobic performance, on markers of muscle damage, and on anabolic/catabolic hormone parameters when both supplements were combined. Due to the different measured outcomes in the studies, the following outcomes have been divided into different groups. The results could be influenced by type of sport, amount of each supplement, and duration of the intervention. Participant characteristics, such as age, gender, ethnicity, body composition, training level, differences in training, nutrition, and health status, may also influence the results.

\subsection{Impact on Sport Performance}

\subsubsection{Strength Performance}

Strength is an essential attribute in sports performance, and describes the capacity to perform any action faster when the same mass is moved [45]. Strength tests require $\mathrm{Cr} / \mathrm{PCr}$ as an energy substrate [46]. Therefore, strength could be enhanced with CrM ingestion, improving muscular performance by an increase of $\mathrm{Cr} / \mathrm{PCr}$ [8]. In addition, $\mathrm{HMB}$ supplementation can also improve strength through an increase of the muscle cross-sectional area [29]. This effect of HMB could be due to an increase of muscle-protein synthesis caused by an up-regulation of the mTOR pathway [27], or by a marked change in oxidative metabolism [29]. Although in one of the two studies analyzed, the strength performance was improved [36]; in the other [40], no changes were found. The main reason for this could be the training status of the participants [47]. However, in the study by Jowko et al., 40 healthy males were measured by 1-RM of different exercises (bench press, behind the neck press, biceps curl, back squat, triceps extension, power clean, and sum of all tests), and it was shown that CrM plus HMB supplementation caused accumulative strength increases [36]. These observations are consistent with, but do not prove, the hypothesis that CrM plus HMB acts through distinct mechanisms, as already described.

\subsubsection{Anaerobic Performance}

The prolonged periods of multiple sprints that occur in different sports (for example soccer, basketball, or rugby) drain muscle glycogen stores, leading to a decrease in energy production and a reduction in the overall work rate during training and/or competition [48] Short-term CrM supplementation has been shown to up-regulate the mRNA content of some genes and proteins involved in glycogen synthesis, producing a change in cellular osmolarity [15]. Moreover, an increase of muscle PCr by CrM supplementation is essential to activities dependent on the PCr energy system [13]. In addition, the $\mathrm{Cr} / \mathrm{PCr}$ shuttle acts as a buffer that reduces LA in anaerobic glycolytic actions [16]. On the other hand, the negative muscle net balance that occurs after resistance exercise must be resolved quickly [49]. To achieve this, leucine must be ingested after exercise. Supplementation with $\mathrm{HMB}$ (leucine is a precursor of HMB) in the 30 minutes post the exercise period could be enough stimulus to produce protein synthesis, facilitating muscle recovery [29].

In relation to this topic, three studies were analyzed $[35,37,39]$. Two of them showed improvements in anaerobic performance [35,37]. Concretely, Faramarzi et al. [35] found a higher anaerobic performance (peak power) in soccer players during the RAST in CR/HMBG with respect to HMBG. This result 
showed an additive effect of combined CrM plus HMB supplementation [35]. Equally, Zajac et al. found, in elite basketballers, better statistical results in CRG and CR/HMBG compared to HMBG and $\mathrm{CON}$ in a triple Wingate test. However, although there were no statistical differences between CRG and CR/HMBG, CR/HMBG showed a better performance in a triple Wingate test [37]. On the other hand, $\mathrm{O}^{\prime}$ Connor et al. did not find changes among supplemented groups after a $60 \mathrm{~s}$ maximal anaerobic capacity test [39]. These results could be related to the high-level anaerobic training status of elite rugby players, and the difficulty in improving results for such athletes [39]. Thus, the combination of CrM plus HMB could help in achieving better results in improving anaerobic performance than taking them individually, however more research is needed to affirm these findings.

\subsubsection{Aerobic Performance}

Aerobic performance is a key factor in long endurance sports (for example long running, cycling, or rowing events) which require maintaining a specific intensity for as long as possible [50]. CrM can improve aerobic capacity by increasing the $\mathrm{Cr} / \mathrm{PCr}$ shuttle that acts like a buffer, lowering the LA concentration at the same intensity [16]. In addition, CrM improves muscle glycogen synthesis [14,15]. HMB can also affect aerobic performance by enhancing aerobic capacity through mitochondrial biogenesis, by activation of PGC-1 $\alpha$ [30]. In this regard, no significant differences were observed among supplemented groups in the only study where aerobic performance was measured by a multistage aerobic capacity test [39]. This result could be due to the fact that the training focused on resistance exercises. Furthermore, the participants were highly trained athletes, who would be closer to their maximum genetic potential compared to untrained subjects [47].

Therefore, these controversial results generate certain doubts about the supplementation of CrM plus HMB and the benefit to aerobic performance. However, future work should be oriented towards exploring their effects on other types of tests and among different groups of athletes.

\subsection{Impact on Body Composition}

To obtain maximum performance, athletes need to have an optimal body composition for the concrete sport practice, with a low-fat mass percentage and optimal skeletal muscle mass [51]. HMB could increase muscle mass [52] by augmenting protein synthesis after an intense training session [27], by activation of mTOR [53]. Equally, it can reduce protein breakdown through reducing the ubiquitin-proteasome system [54] and by increasing the GH-IGF-I axis [55]. In addition, HMB could augment fat oxidation, improving mitochondrial biogenesis by activation of PGC- $1 \alpha$, and thereby, lowering the fat mass percentage [30]. On the other hand, CrM supplementation has been proposed to increase muscle mass by increasing osmotic pressure in muscle, which increases the water content of the muscle [56-58], which in turn promotes glycogen synthesis [14,15].

The mixture of CrM plus HMB could augment FFM or LBM more than taking them in an isolated way, as the study by Zajac et al. [37] demonstrated. However, in the study by Jowko et al. [36], no changes were found. FM had also can be reduced [37] or unchanged [36]. In this sense, Zajac et al. [37] obtained an interesting result, given that mixed supplementation combined with three full-body resistance training sessions per week appeared to help to increase BM and decrease FM. HMBG decreased BF in comparison to CRG and CONG, and CRG increased BM (without increasing FM) in contrast to HMBG and CONG, showing an accumulative effect in terms of enhancing body composition when they were ingested together. Moreover, in the investigation by $\mathrm{O}^{\prime}$ Connor et al. [40], body composition parameters measured (BM, sum of six skinfolds, arm girth (relaxed), arm girth (fixed), chest girth, waist girth, hip girth, and thigh girth) were not changed when combined with three full body resistance training sessions and one speed/power session per week. These results might be explained by the high training level of the participants. Therefore, the combination of CrM plus HMB could have an additive effect due to the performance of different energetic pathways. 


\subsection{Impact on markers of Muscle Damage and Hormone Status}

\subsubsection{Markers of Exercise-Induced Muscle Damage}

The markers of exercise-induced muscle damage markers that were measured, LDH, CK, and LA, could be predictors of training intensity. Therefore, the activity of these markers is potentially useful, not as a marker of impending overtraining, but as a means of identifying a state of recent muscle damage or temporary over-reaching [59]. When CrM and HMB are taken in an isolated manner CrM can reduce LA [60], LDH [12], and CK [12] levels, and HMB can also decrease LA [20], CK [61], and $\mathrm{LDH}$ [61] levels after training. On the other hand, when the supplements are taken together, they do not show positive effects on CK [35,36,38], LDH [35], and LA [37,39] levels. Concretely, in the study by Jowko et al. [36], CK levels remained elevated following three weeks of CrM/HMBG supplementation. This could explain a decrease of protein degradation via HMB [62], an increase of LBM and improvements in strength achieved by this supplementation. This study [36] did not show an additive effect of HMB and CrM; quite the opposite, with CrM impairing HMB's results. In summary, the results of the studies showed no better effect from combining both supplements on markers of muscle damage markers compared to individual intake.

\subsubsection{Anabolic/Catabolic Hormones}

Monitoring testosterone and cortisol could provide insight into an athlete's recovery/readiness, and could be a tool to program daily volume/intensity of training [63]. While testosterone is an anabolic and anticatabolic hormone that indicates the degree of endogenous regeneration, cortisol indicates accumulated stress [63].

HMB ingestion can reduce blood cortisol levels [64] and CrM can increase testosterone levels [32,33] when they are taken individually. There was only one study that analyzed hormone status when the mixed supplements were ingested [38], and it showed no differences in cortisol or testosterone levels after six weeks of supplementation. It is difficult to obtain changes in this parameter in few time (6 weeks) [65], however, and more studies are needed to understand the effect of CrM and HMB in combination on anabolic/catabolic hormone responses.

\subsection{Strengths, Limitations and Future Research}

In this systematic review, we analyzed six studies [35-40] considering different sport modalities (rugby, basketball, and soccer), outcomes, and supplementation duration. Moreover, the tests used to measure strength, anaerobic performance, and aerobic performance were completely different. These differences resulted in difficulties in comparing the different outcomes of the studies. Furthermore, not all the studies analyzed the effect of the supplementation mixture on different groups. Therefore, the results of this systematic review should be treated with caution due to the small number of research works available to include that are relevant to this area. Accordingly, more studies with similar measurement methodologies are needed in order to determine the efficacy of mixing CrM plus HMB for improving sports performance, and in order to understand the potential additive effect of this combination.

\section{Conclusions}

In summary, the main results of this systematic review seem to indicate that the combination of 3-10 g/day of CrM plus $3 \mathrm{~g} /$ day HMB for 1-6 weeks could produce improvements in strength performance, anaerobic performance, and for 4 weeks in body composition (increasing FFM and decreasing FM), that may exceed the effects of taking them in an isolated way. However, no significant results relating to markers of exercise-induced muscle damage and anabolic/catabolic hormone status were found when both supplements were combined. 
Author Contributions: J.F.-L. and J.M.-A. conceived and designed the review, analyzed and interpreted the data, drafted the paper, and approved the final version submitted for publication. J.C.-G. analyzed and interpreted the data, critically reviewed the paper, and approved the final version submitted for publication. A.C., A.C.-G., and P.L.-G. critically reviewed the paper and approved the final version submitted for publication.

Funding: Funding information is not applicable/no funding was received.

Acknowledgments: The authors thank the Foundation Institute of Studies of Health Sciences of Castilla y León (IECSCYL) for their collaboration on infrastructure and computer support.

Conflicts of Interest: The authors declare no conflict of interest.

\section{References}

1. Thomas, D.T.; Erdman, K.A.; Burke, L.M. Position of the Academy of Nutrition and Dietetics, Dietitians of Canada, and the American College of Sports Medicine: Nutrition and Athletic Performance. J. Acad. Nutr. Diet. 2016, 116, 501-528. [CrossRef] [PubMed]

2. Australian Institute of Sport. Available online: https://www.sportaus.gov.au/_data/assets/pdf_file/0004/ 698557/AIS_Sports_Supplement_Framework_2019.pdf (accessed on 19 March 2019).

3. Maganaris, C.N.; Maughan, R.J. Creatine supplementation enhances maximum voluntary isometric force and endurance capacity in resistance trained men. Acta Physiol. Scand. 1998, 163, 279-287. [CrossRef] [PubMed]

4. Chwalbinska-Moneta, J. Effect of creatine supplementation on aerobic performance and anaerobic capacity in elite rowers in the course of endurance training. Int. J. Sport Exerc. Metab. 2003, 13, 173-183. [CrossRef]

5. Bazzucchi, I.; Felici, F.; Sacchetti, M. Effect of short-term creatine supplementation on neuromuscular function. Int. J. Sport Nutr. Exerc. Metab. 2009, 41, 1934-1941. [CrossRef] [PubMed]

6. Kreider, R.B.; Ferreira, M.; Wilson, M.; Grindstaff, P.; Plisk, S.; Reinardy, J.; Cantler, E.; Almada, A.L. Effects of creatine supplementation on body composition, strength, and sprint performance. Med. Sci. Sports Exerc. 1998, 30, 73-82. [CrossRef] [PubMed]

7. Urbanski, R.L.; Vincent, W.J.; Yaspelkis, B.B. Creatine supplementation differentially affects maximal isometric strength and time to fatigue in large and small muscle groups. Int. J. Sport Nutr. 1999, 9, 136-145. [CrossRef] [PubMed]

8. Becque, M.; Lochmann, J.; Melrose, D. Effects of oral creatine supplementation on muscular strength and body composition. Phys. Fit. Perform. 2000, 32, 654-658. [CrossRef] [PubMed]

9. Warber, J.P.; Tharion, W.J.; Patton, J.F.; Champagne, C.M.; Mitotti, P.; Lieberman, H.R. The effect of creatine monohydrate supplementation on obstacle course and multiple bench press performance. J. Strength Cond. Res. 2002, 16, 500-508.

10. Kutz, M.R.; Gunter, M.J. Creatine Monohydrate Supplementation on Body Weight and Percent Body Fat. J. Strength Cond. Res. 2003, 17, 817-821.

11. Cooke, M.B.; Rybalka, E.; Williams, A.D.; Cribb, P.J.; Hayes, A. Creatine supplementation enhances muscle force recovery after eccentrically-induced muscle damage in healthy individuals. J. Int. Soc. Sports Nutr. 2009, 6, 13. [CrossRef]

12. Bassit, R.; Pinheiro, C.; Vitzel, K.; Sproesser, A.; Silveira, L.; Curi, R. Effect of short-term creatine supplementation on markers of skeletal muscle damage after strenuous contractile activity. Eur. J. Appl. Physiol. 2010, 108, 945-955. [CrossRef] [PubMed]

13. Kerksick, C.M.; Arent, S.; Schoenfeld, B.J.; Stout, J.R.; Campbell, B.; Wilborn, C.D.; Taylor, L.; Kalman, D.; Smith-Ryan, A.E.; Kreider, R.B.; et al. International society of sports nutrition position stand: Nutrient timing. J. Int. Soc. Sports Nutr. 2017, 14, 1-12. [CrossRef] [PubMed]

14. Roberts, P.A.; Fox, J.; Peirce, N.; Jones, S.W.; Casey, A.; Greenhaff, P.L. Creatine ingestion augments dietary carbohydrate mediated muscle glycogen supercompensation during the initial $24 \mathrm{~h}$ of recovery following prolonged exhaustive exercise in humans. Amino Acids 2016, 48, 1831-1842. [CrossRef] [PubMed]

15. Van Loon, L.J.; Murphy, R.; Oosterlaar, A.M.; Cameron-Smith, D.; Hargreaves, M.; Wagenmakers, A.J.M.; Snow, R. Creatine supplementation increases glycogen storage but not GLUT-4 expression in human skeletal muscle. Clin. Sci. 2004, 106, 99-106. [CrossRef] [PubMed]

16. Saks, V.A.; Kongas, O.; Vendelin, M.; Kay, L. Role of the creatine/phosphocreatine system in the regulation of mitochondrial respiration. Acta Physiol. Scand. 2000, 168, 635-641. [CrossRef] 
17. Chilibeck, P.; Kaviani, M.; Candow, D.; Zello, G.A. Effect of creatine supplementation during resistance training on lean tissue mass and muscular strength in older adults: a meta-analysis. Open Access J. Sport Med. 2017, 8, 213-226. [CrossRef]

18. Durkalec-Michalski, K.; Jeszka, J. The efficacy of a $\beta$-hydroxy- $\beta$-methylbutyrate supplementation on physical capacity, body composition and biochemical markers in elite rowers: A randomised, double-blind, placebo-controlled crossover study. J. Int. Soc. Sports Nutr. 2015, 12, 31. [CrossRef]

19. Vukovich, M.D.; Dreifort, G.D. Effect of beta-hydroxy beta-methylbutyrate on the onset of blood lactate accumulation and VO2 peak in endurance-trained cyclists. J. Strength Cond. Res. 2001, 15, 491-497.

20. Durkalec-Michalski, K.; Jeszka, J.; Podgórski, T. The effect of a 12-week beta-hydroxy-beta-methylbutyrate (HMB) supplementation on highly-trained combat sports athletes: A randomised, double-blind, placebo-controlled crossover study. Nutrients 2017, 9, 753. [CrossRef]

21. Asadi, A.; Arazi, H.; Suzuki, K. Effects of $\beta$-hydroxy- $\beta$-methylbutyrate-free acid supplementation on strength, power and hormonal adaptations following resistance training. Nutrients 2017, 9, 1316. [CrossRef]

22. Meckel, Y.; Cooper, D.M.; Eliakim, A.; Nemet, D. The effect of HMB supplementation on body composition, fitness, hormonal and inflammatory mediators in elite adolescent volleyball players: A prospective randomized, double-blind, placebo-controlled study. Eur. J. Appl. Physiol. 2011, 111, 2261-2269.

23. Wilson, J.M.; Lowery, R.P.; Joy, J.M.; Andersen, J.C.; Wilson, S.M.C.; Stout, J.R.; Duncan, N.; Fuller, J.C.; Baier, S.M.; Naimo, M.A.; et al. The effects of 12 weeks of beta-hydroxy-beta-methylbutyrate free acid supplementation on muscle mass, strength, and power in resistance-trained individuals: A randomized, double-blind, placebo-controlled study. Eur. J. Appl. Physiol. 2014, 114, 1217-1227. [CrossRef] [PubMed]

24. Thomson, J.; Watson, P.; Rowlands, D. Effects of nine weeks of b-hydroxy-b-methylbutyrate supplementation on strength and body composition in resistance trained men. J. Strength Cond. Res. 2009, 23, 827-835. [CrossRef] [PubMed]

25. Durkalec-Michalski, K.; Jeszka, J. The effect of $\beta$-hydroxy- $\beta$-methylbutyrate on aerobic capacity and body composition in trained athletes. J. Strength Cond. Res. 2016, 30, 2617-2626. [CrossRef] [PubMed]

26. Ferreira, H.; Gill, P.; Fernandes Filho, J.; Fernandes, L. Effects of 12-weeks of supplementation with $\beta$-hydroxy- $\beta$-methylbutyrate-Ca (HMB-Ca) on athletic performance. J. Exerc. Physiol. 2015, 18, 84-94.

27. Wilson, J.M.; Fitschen, P.J.; Campbell, B.; Wilson, G.J.; Zanchi, N.; Taylor, L.; Wilborn, C.; Kalman, D.S.; Stout, J.R.; Hoffman, J.R.; et al. International Society of Sports Nutrition Position Stand: Beta-hydroxy-beta-methylbutyrate (HMB). J. Int. Soc. Sports Nutr. 2013, 10, 6. [CrossRef] [PubMed]

28. Di Camillo, B.; Eduati, F.; Nair, S.K.; Avogaro, A.; Toffolo, G.M. Leucine modulates dynamic phosphorylation events in insulin signaling pathway and enhances insulin-dependent glycogen synthesis in human skeletal muscle cells. BMC Cell Biol. 2014, 15, 1-9. [CrossRef]

29. Pinheiro, C.; Gerlinger-Romero, F.; Guimarães-Ferreira, L.; de Souza, A.J.; Vitzel, K.; Nachbar, R.; Nunes, M.; Curi, R. Metabolic and functional effects of beta-hydroxy-beta-methylbutyrate (HMB) supplementation in skeletal muscle. Eur. J. Appl. Physiol. 2012, 112, 2531-2537. [CrossRef]

30. He, X.; Duan, Y.; Yao, K.; Li, F.; Hou, Y.; Wu, G.; Yin, Y. $\beta$-Hydroxy- $\beta$-methylbutyrate, mitochondrial biogenesis, and skeletal muscle health. Amino Acids 2016, 48, 653-664. [CrossRef]

31. Park, B.S.; Henning, P.C.; Grant, S.C.; Lee, W.J.; Lee, S.R.; Arjmandi, B.H.; Kim, J.S. HMB attenuates muscle loss during sustained energy deficit induced by calorie restriction and endurance exercise. Metabolism 2013, 62, 1718-1729. [CrossRef]

32. Vatani, D.S.; Faraji, H.; Soori, R.; Mogharnasi, M. The effects of creatine supplementation on performance and hormonal response in amateur swimmers. Sci. Sport 2011, 26, 272-277. [CrossRef]

33. Arazi, H.; Rahmaninia, F.; Hosseini, K.; Asadi, A. Effects of short term creatine supplementation and resistance exercises on resting hormonal and cardiovascular responses. Sci. Sport 2015, 30, 105-109. [CrossRef]

34. Wilson, G.J.; Wilson, J.M.; Manninen, A.H. Effects of beta-hydroxy-beta-methylbutyrate (HMB) on exercise performance and body composition across varying levels of age, sex, and training experience: A review. Nutr. Metab. 2008, 5, 1-17. [CrossRef] [PubMed]

35. Faramarzi, M.; Nuri, R.; Banitalebi, E.; Sciences, S. The effect of short-term combination of HMB (beta-hydroxy-beta-methylbutyrate) and creatine supplementation on anaerobic performance and muscle injury markers in soccer players. Braz. J. Biomotricity 2009, 3, 366-375. 
36. Jówko, E.; Ostaszewski, P.; Jank, M.; Sacharuk, J.; Zieniewicz, A.; Wilczak, J.; Nissen, S. Creatine and $\beta$-hydroxy- $\beta$-methylbutyrate (HMB) additively increase lean body mass and muscle strength during a weight-training program. Nutrition 2001, 17, 558-566. [CrossRef]

37. Zajac, A.; Waskiewicz, Z.; Poprzecki, S.; Cholewa, J. Effects of creatine and HMß supplementation on anaerobic power and body composition in basketball players. J. Hum. Kinet. 2003, 10, 95-108.

38. Crowe, M.J.; O'Connor, D.M.; Lukins, J.E. The effects of $\beta$-hydroxy- $\beta$-methylbutyrate (HMB) and $\mathrm{HMB} /$ creatine supplementation on indices of health in highly trained athletes. Int. J. Sport Nutr. Exerc. Metab. 2003, 13, 184-197. [CrossRef]

39. O'Connor, D.M.; Crowe, M.J. Effects of beta-hydroxy-beta-methylbutyrate and creatine monohydrate supplementation on the aerobic and anaerobic capacity of highly trained athletes. J. Sports Med. Phys. Fitness 2003, 43, 64-68.

40. O'Connor, D.M.; Crowe, M.J. Effects of six weeks of beta-hydroxy-beta-methylbutyrate (HMB) and $\mathrm{HMB} /$ creatine supplementation on strength, power, and anthropometry of highly trained athletes. J. Strength Cond. Res. 2007, 21, 419-423. [CrossRef]

41. Moher, D.; Liberati, A.; Tetzlaff, J.; Altman, D.G. Academia and clinic annals of internal medicin preferred reporting items for systematic reviews and meta-analyses. Ann. Intern. Med. 2009, 151, 264-269.

42. Higgins, J.; Green, S. Cochrane Handbook for Systematic Reviews of Interventions; John Wiley \& Sons: Chichester, UK, 2008.

43. Chulvi-Medrano, I.; Picon-Martinez, M.; Garcia-Jaen, M.; Manuel Cortell-Tormo, J.; Alakhdar, Y.; Laurentino, G. Neuromuscular adaptations after blood flow restriction training combined with nutritional supplementation: A preliminary study. Montenegrin J. Sport Sci. Med. 2019, 8, 37-42. [CrossRef]

44. Miramonti, A.; Stout, J.; Fukuda, D.; Robinson, E.; Wang, R.; La Monica, M.; Hoffman, J. The effects off four weeks of high intensity interval training and $\beta$-hydroxy- $\beta$-methylbutyric free acid on the onset of neuromuscular fatigue. J. Strength Cond. Res. 2015, 30, 626-634. [CrossRef] [PubMed]

45. Folland, J.P.; Williams, A.G. The adaptations to strength training: Morphological and neurological contributions to increased strength. Sport Med. 2007, 37, 145-168. [CrossRef] [PubMed]

46. Kreider, R.B.; Kalman, D.S.; Antonio, J.; Ziegenfuss, T.N.; Wildman, R.; Collins, R.; Candow, D.G.; Kleiner, S.M.; Almada, A.L.; Lopez, H.L. International Society of Sports Nutrition position stand: Safety and efficacy of creatine supplementation in exercise, sport, and medicine. J. Int. Soc. Sports Nutr. 2017, 14, 1-18. [CrossRef] [PubMed]

47. Spurway, N.; MacLaren, D. The Physiology of Training: Advances in Sport and Exercise Science Series; Elsevier Health Sciences: Philadelphia, PA, USA, 2006.

48. Williams, C.; Rollo, I. Carbohydrate nutrition and team sport performance. Sport.Med. 2015, 45, 13-22. [CrossRef]

49. Phillips, S.M.; Tipton, K.D.; Ferrando, A.A.; Wolfe, R.R. Resistance training reduces the acute exercise-induced increase in muscle protein turnover. Am. J. Physiol. 1999, 276, E118-E124. [CrossRef]

50. Córdova Martínez, A.; Fernández-Lázaro, D.; Mielgo-Ayuso, J.; Seco Calvo, J.; Caballero García, A. Effect of magnesium supplementation on muscular damage markers in basketball players during a full season. Magnes. Res. 2017, 30, 61-70. [CrossRef]

51. Martin, D.T.; McLean, B.; Trewin, C.; Lee, H.; Victor, J.; Hahn, A.G. Physiological characteristics of nationally competitive female road cyclists and demands of competition. Sport Med. 2001, 31, 469-477. [CrossRef]

52. Nissen, S.L.; Sharp, R.L. Effect of dietary supplements on lean mass and strength gains with resistance exercise: A meta-analysis. J. Appl. Physiol. 2003, 94, 651-659. [CrossRef]

53. Kornasio, R.; Riederer, I.; Butler-Browne, G.; Mouly, V.; Uni, Z.; Halevy, O. $\beta$-hydroxy- $\beta$-methylbutyrate (HMB) stimulates myogenic cell proliferation, differentiation and survival via the MAPK/ERK and PI3K/Akt pathways. Biochim. Biophys. Acta Mol. Cell Res. 2009, 1793, 755-763. [CrossRef]

54. Smith, H.J.; Mukerji, P.; Tisdale, M.J. Attenuation of proteasome-induced proteolysis in skeletal muscle by $\beta$-hydroxy- $\beta$-methylbutyrate in cancer-induced muscle loss. Cancer Res. 2005, 65, 277-283. [PubMed]

55. Dehoux, M.; Van Beneden, R.; Pasko, N.; Lause, P.; Verniers, J.; Underwood, L.; Ketelslegers, J.M.; Thissen, J.P. Role of the insulin-like growth factor I decline in the induction of atrogin-1/MAFbx during fasting and diabetes. Endocrinology 2004, 145, 4806-4812. [CrossRef] [PubMed] 
56. Stone, M.; Sanborn, K.; Smith, L.; O’Bryant, H.; Hoke, T.; Utter, A.; Johnson, R.; Boros, R.; Hruby, J.; Pierce, K.; et al. Effects of in-season ( 5 weeks) creatine and pyruvate supplementation on anaerobic performance and body composition in American football players. Int. J. Sport Nutr. 1999, 9, 146-165. [CrossRef] [PubMed]

57. Deminice, R.; Rosa, F.T.; Pfrimer, K.; Ferrioli, E.; Jordao, A.A.; Freitas, E. Creatine Supplementation Increases Total Body Water in Soccer Players: A Deuterium Oxide Dilution Study. Int. J. Sports Med. 2016, 37, 149-153. [CrossRef] [PubMed]

58. Powers, M.E.; Arnold, B.L.; Weltman, A.L.; Perrin, D.H.; Mistry, D.; Kahler, D.M.; Kraemer, W.; Volek, J. Creatine Supplementation Increases Total Body Water Without Altering Fluid Distribution. J. Athl. Train. 2003, 38, 44-50. [PubMed]

59. Gleeson, M. Biochemical and immunological markers of over-training. J. Sport Sci. Med. 2002, 1, 31-41.

60. Oliver, J.; Joubert, D.; Martin, S.; Crouse, S. Oral creatine supplementation's decrease of blood lactate during exhaustive, incremental cycling. Int. J. Sport Nutr. Exerc. Metab. 2013, 23, 252-258. [CrossRef]

61. Knitter, A.E.; Panton, L.; Rathmacher, J.A.; Petersen, A.; Sharp, R. Effects of $\beta$-hydroxy- $\beta$-methylbutyrate on muscle damage after a prolonged run. J. Appl. Physiol. 2000, 89, 1340-1344. [CrossRef]

62. Nissen, S.; Sharp, R.; Ray, J.; Rathmacher, J.A.; Rice, D.; Fuller, J.C.; Connelly, A.S.; Abumrad, N. Effect of leucine metabolite $\beta$-hydroxy- $\beta$-methylbutyrate on muscle metabolism during resistance-exercise training. J. Appl. Physiol. 1996, 81, 2095-2104. [CrossRef]

63. Mielgo-Ayuso, J.; Zourdos, M.; Urdampilleta, A.; Calleja-González, J.; Seco, J.; Córdova, A. Relationship of long-term macronutrients intake on anabolic-catabolic hormones in female elite volleyball players. Nutr. Hosp. 2017, 34, 1155-1162. [CrossRef]

64. Tinsley, G.M.; Givan, A.H.; Graybeal, A.J.; Villarreal, M.I.; Cross, A.G. $\beta$-Hydroxy $\beta$-methylbutyrate free acid alters cortisol responses, but not myofibrillar proteolysis, during a 24-h fast. Br. J. Nutr. 2018, 119, 517-526. [CrossRef] [PubMed]

65. Häkkinen, K.; Pakarinen, A.; Alén, M.; Komi, P.V. Serum hormones during prolonged training of neuromuscular performance. Eur. J. Appl. Physiol. Occup. Physiol. 1985, 53, 287-293. [CrossRef] [PubMed]

(C) 2019 by the authors. Licensee MDPI, Basel, Switzerland. This article is an open access article distributed under the terms and conditions of the Creative Commons Attribution (CC BY) license (http://creativecommons.org/licenses/by/4.0/). 